\title{
Reflection and Propagation of Electromagnetic Wave in Time-varying Medium
}

\author{
Bingkang Chen \\ College of Information, Linyi University, Linyi 276000, China \\ Tel: 86-539-820-7638 E-mail: chenbingkang@lyu.edu.cn \\ Xufei Lu \\ Linyi Center for Disease Control and Prevention, Linyi 276000, China \\ Tel: 86-539-831-4790Ｅ-mail: xufeilu001@163.com
}

Received: April 6, 2011

Accepted: April 27, 2011

doi:10.5539/mas.v5n4p243

The research is financed by the Doctor Scientific Research Fund (No. Bs08004). (Sponsoring information)

\begin{abstract}
The reflection of plane electromagnetic wave on the interface between time-varying medium and time-invariant medium and the propagation of plane electromagnetic wave in time-varying medium are studied in this article. The medium which changes with time as an exponential function is observed and the analytical solution of the electromagnetic wave in such medium is obtained by the method of separation variables. The transient reflection coefficient on the surface of the time-varying medium and the transient transmission coefficient in time-varying medium are obtained for the first time by taking the limit for time increment. The validity of the solution is testified by three approaches. The propagation characteristics are discussed by the example in different areas.
\end{abstract}

Keywords: Time-varying medium, Method of separation variables, Finite-different time-domain (FDTD), Wave splitting

\section{Introduction}

The time-varying medium means the medium which parameters $\varepsilon, \mu$, or $\sigma$ change with the time. Many scholars have studied the propagation character in the time-varying medium (F. R. Morenthaler, 1958, P. 167-172 \& R. L. Fante, 1971, P. 417-424 \& A.G. Nerukh, 2004, P. 1408-1419 \& Ruiz T M, 1978, P. 358-361 \& Ren, 2003, P. 18-22 \& Y. Zhang, 2005, P. 446-449 \& B. K. Chen, 2006, P. 595-598 \& B. K. Chen, 2009, P. 68-76). And the physical phenomenon occurred in the propagation process that the plane electromagnetic wave encounters the time-varying medium should be noticed, which involves many problems, such as the reflection on the interface, the quantity entering into the time-varying medium, and the physical character when the wave propagates in the time-varying medium. Fante (R. L. Fante, 1971, P. 417-424) studied the propagation that the plane wave transmits into the time-varying medium by the inclined way, but the studied time-varying medium changed step by step with time, and for the medium continually changed with the time, Fante used this medium with step change of time to approach the researched continual time-varying medium, and the obtained solution was the approximate solution.

To seek the exact solutions about the reflection and transmission of the electromagnetic wave on the interface between the time-varying medium and the time-invariant medium is always the dynamic in the researches. In this article, the methods of the separation variable and the mathematic limit are adopted to study the time-varying medium changing continually with the time, and the exact solution of the electromagnetic wave is obtained aiming at the concrete time-varying form, including the expression of the electromagnetic field in the time-varying medium and the reflection and propagation parameters on the interface between two mediums.

Generally, the time-varying forms of medium are varied, but not all time-varying forms have analytic solutions. Because of the possibility of the analytic solution and the conductivity of lunar rock which can be regarded as the double-exponential function of time (Ouyang, 2005), to study the change of the medium parameters with the exponential form has both practical meaning and theoretical meaning. For other time-varying forms of medium, if the analytic solution could not be obtained, it can be solved by the numerical method.

In the second part of this article, the problem model is established, and the variable separation method is used to separate the second-order differential equation of the electromagnetic field in the time-varying medium into the differential equation only to the space variable and the differential equation only to the time variable, and obtain the exact solution of the field when only knowing the concrete changing form of the time-varying medium and the initial conditions of the field. In the third part of this article, the correctness of the solution is validated from three aspects. First, through taking the limit of the time-varying parameters in the time-varying medium, the time-varying medium will be changed to the time-invariant medium, and the corresponding expression of the 
electromagnetic wave is changed to the expression of the wave in the time-invariant medium. Second, by changing the gradually changing medium parameters as the mutation parameters, the obtained solution will be changed to the expression of the field in the time-variant medium. Third, the FDTD method is used to study the propagation of the electromagnetic wave in the time-varying medium and in the time-invariant medium, and the research result shows that the results obtained from these two methods could be fitted well. The fourth part of this article will discuss the changing rule of the reflection wave, the propagation wave, and the distribution figure of corresponding parameters based on examples.

\section{Model and its solution}

Supposed that the space is filled by the medium with the even and isotropy dielectric constant $\varepsilon_{0}$, the magnetic conductivity $\mu_{0}$, and the conductivity $\sigma=0$ when $t<t_{1}$. From the time of $t=t_{1}$, the medium in the space of $z>0$ evenly changes according to the rule that the dielectric constant is $\varepsilon_{0} \varepsilon(t)$ and the conductivity is $\sigma(t)$, and the medium in the space of $z<0$ is invariant. The geometric description of the problem is seen in Figure 1.

Without loss of generality, when $t<t_{1}$, suppose that the plane electromagnetic wave which frequency is $\omega$ propagates along the direction of $\hat{\boldsymbol{z}}$, its expression of electromagnetic field is

$$
\begin{aligned}
& \boldsymbol{E}^{\boldsymbol{i}}=\hat{\boldsymbol{x}} E_{\mathbf{0}} \exp \left[\mathrm{i}\left(\omega t-k_{0} z\right)\right] \quad t<t_{1} \\
& \boldsymbol{H}^{\boldsymbol{i}}=\hat{\boldsymbol{y}} E_{\mathbf{0}} \exp \left[\mathrm{i}\left(\omega \omega-k_{0} z\right)\right] / \eta_{\mathbf{0}} \quad t<t_{1}
\end{aligned}
$$

, where, $\hat{\boldsymbol{x}}$ and $\hat{\boldsymbol{y}}$ are unit vectors, $E_{\mathbf{0}}$ is the value of initial field intensity, $k_{0}$ is the wave amount along the director of $z, \eta_{0}=\sqrt{\mu_{0} / \varepsilon_{0}}$ is the characteristic impedance of air. When $t>t_{1}$, the boundary of the medium is the plane of $z=0$, and the space of $z>0$ is the time-varying medium space, and the space of $z<0$ is the time-invariant medium space.

For the electromagnetic wave in the Area One, the incident wave emits the plane of $z=0$ from the Area One, and because the tangential component of the electric field is continual on two sides of the boundary, so the electric fields of the reflection wave and the transmission wave only have the component of the direction of $x$. In the same way, the reflection electromagnetic field components in the Area One are

$$
\begin{aligned}
& E_{x}^{r}=E_{0} \Gamma(t) \exp \left[\mathrm{i}\left(\omega \omega+k_{0} z\right)\right] t>t_{1} \\
& H_{y}^{r}=-E_{0} \Gamma(t) \exp \left[\mathrm{i}\left(\omega t+k_{0} z\right)\right] / \eta_{0} t>t_{1}
\end{aligned}
$$

, where, $\Gamma(t)$ is the reflection coefficient of the interface at the time of $t$.

From (1a), (1b), (2a), and (2b), the components of the total electromagnetic field in the Area One at the time of $t$ can be obtained.

$$
\begin{aligned}
& E_{x}^{\text {tol }}=E_{x}^{i}+E_{x}^{r} \\
& H_{y}^{\text {tol }}=H_{y}^{i}+H_{y}^{r}
\end{aligned}
$$

To seek the transient reflection coefficient and the transient propagation coefficient of the wave on the interface of $z=0$, the method of taking the limit for the time-varying increment is used to analyzed the data. $t$ is the initial time, and $\Delta t$ is the very small quantity, so in the time period from $t$ to $t+\Delta t$, the medium parameters in the Area Two can be approximately regarded as the constant, such as $\varepsilon_{0} \varepsilon(t+\Delta t / 2)$ and $\sigma(t+\Delta t / 2)$, for the electromagnetic field $\boldsymbol{E}^{T}$ in the period from $t$ to $t+, \boldsymbol{H}^{T}$ satisfies

$$
\begin{aligned}
\nabla \times \boldsymbol{E}^{\boldsymbol{T}} & =-\mu_{0} \frac{\partial}{\partial t} \boldsymbol{H}^{\boldsymbol{T}} \\
\nabla \times \boldsymbol{H}^{\boldsymbol{T}} & =\sigma(t+\Delta t / \mathbf{2}) \boldsymbol{E}^{\boldsymbol{T}}+\varepsilon_{0} \varepsilon(t+\Delta t / \mathbf{2}) \frac{\partial}{\partial t} \boldsymbol{E}^{\boldsymbol{T}}
\end{aligned}
$$

Unite (4a) with (4b),

$$
\begin{aligned}
& \boldsymbol{E}^{T}=\hat{\boldsymbol{x}} T(t+\Delta t / 2) E_{0} \exp (-\alpha(t+\Delta t / 2) z) \times \exp [\mathrm{i}(\omega t-\beta(t+\Delta t / 2) z)] \\
& \boldsymbol{H}^{\boldsymbol{T}}=\hat{\boldsymbol{y}} T(t+\Delta t / 2) E_{0} \exp (-\alpha(t+\Delta t / 2) z) \times \exp [\mathrm{i}(\omega t-\beta(t+\Delta t / 2) z)] / \eta_{1}(t+\Delta t / 2)
\end{aligned}
$$

$$
\text { , where, } T(t+\Delta t / 2) \text { is the propagation coefficient of wave in the period from } t \text { to } t+\Delta t \text {, }
$$


$\eta_{1}(t+\Delta t / 2)=\sqrt{\frac{\mu_{0}}{\varepsilon_{0} \varepsilon(t+\Delta t / 2)-i \sigma(t+\Delta t / 2) / \omega}}$ is the characteristic impedance of wave in the period from $t$ to $t+\Delta t, \alpha(t+\Delta t / \mathbf{2})=\omega \sqrt{\frac{\mu_{0} \varepsilon_{0} \varepsilon(t+\Delta t / 2)}{2}} \times\left[\sqrt{1+\left(\frac{\sigma(t+\Delta t / 2)}{\omega \varepsilon_{0} \varepsilon(t+\Delta t / 2)}\right)^{2}}-1\right]^{1 / 2}$ is the attenuation constant of wave in the period from $t$ to $t+\Delta t$, $\beta(t+\Delta t / 2)=\omega \sqrt{\frac{\mu_{0} \varepsilon_{0} \varepsilon(t+\Delta t / 2)}{2}} \times\left[\sqrt{1+\left(\frac{\sigma(t+\Delta t / 2}{\omega \varepsilon_{0} \varepsilon(t+\Delta t / 2)}\right)^{2}}+1\right]^{1 / 2}$ is the phase constant of wave in the period from $t$ to $t+\Delta t$.

When $\Delta t$ is close to 0 , the transient parameters at the time of $t$ are

$\eta_{1}(t)=\lim _{\Delta t \rightarrow 0} \eta_{1}(t+\Delta t)$

$\alpha(t)=\lim _{\Delta \rightarrow 0} \alpha(t+\Delta t)$

$\beta(t)=\lim _{\Delta t \rightarrow 0} \beta(t+\Delta t)$

The electromagnetic field entering into the time-varying medium at the time of $t$ from (5a) and (5b), from the boundary condition $(z=0)$, at the time of $t$,

$E_{0}+E_{0} \Gamma(t)=T(t) E_{0}$

$\frac{E_{0}}{\eta_{0}}-\frac{E_{0}}{\eta_{0}} \Gamma(t)=\frac{T(t)}{\eta_{1}(t)} E_{0}$

, and the solutions are

$\Gamma(t)=\frac{\eta_{1}(t)-\eta_{0}}{\eta_{1}(t)+\eta_{0}}$

$T(t)=\frac{2 \eta_{1}(t)}{\eta_{1}(t)+\eta_{0}}$

The formula (6) and the formula (7) respectively are the transient reflection coefficient and the transient propagation coefficient at the time of $t$ when the wave enters into the time-varying medium from the time-variant medium.

For the electromagnetic wave in the Area Two, the Maxwell equation set of the electromagnetic field when $t>t_{1}$ is

$\nabla \times \boldsymbol{E}^{T}=-\mu_{0} \frac{\partial}{\partial t} \boldsymbol{H}^{T}$

$\nabla \times \boldsymbol{H}^{T}=\sigma(t) \boldsymbol{E}^{T}+\varepsilon_{0} \frac{\partial}{\partial t} \varepsilon(t) \boldsymbol{E}^{T}$

, where, $\boldsymbol{E}^{\boldsymbol{T}}$ and $\boldsymbol{H}^{\boldsymbol{T}}$ respectively denotes the electric-field vector and the magnetic intensity vector in the Area Two. The scalar equation which the electric-field intensity component satisfies from (8a) and (8b) is

$\frac{\partial^{2} E_{x}^{T}}{\partial z^{2}}=\mu_{0}\left(E_{x}^{T} \frac{\mathrm{d} \sigma(t)}{\mathrm{d} t}+\sigma(t) \frac{\partial E_{x}^{T}}{\partial t}\right)+\frac{1}{c^{2}}\left(E_{x}^{T} \frac{\mathrm{d}^{2} \varepsilon(t)}{\mathrm{d} t^{2}}+2 \frac{\mathrm{d} \varepsilon(t)}{\mathrm{d} t} \frac{\partial E_{x}^{T}}{\partial t}+\varepsilon(t) \frac{\partial^{2} E_{x}^{T}}{\partial t^{2}}\right)$

, where, $c=1 / \sqrt{\varepsilon_{0} \mu_{0}}$ is the light speed in the vacuum.

By the variable separation, supposed that $E_{x}^{T}=E_{1}(z) E_{2}(t)$, substitute it into the formula (9),

$\frac{1}{E_{1}} \frac{\mathrm{d}^{2} E_{1}}{\mathrm{~d} z^{2}}=\frac{\mu_{0}}{E_{2}}\left(\frac{\mathrm{d} \sigma(t)}{\mathrm{d} t}+\sigma(t) \frac{\mathrm{d} E_{2}}{\mathrm{~d} t}\right)+\frac{1}{c^{2}}\left(\frac{\mathrm{d}^{2} \varepsilon(t)}{\mathrm{d} t^{2}}+\frac{2}{E_{2}} \frac{\mathrm{d} \varepsilon(t)}{\mathrm{d} t} \frac{\mathrm{d} E_{2}}{\mathrm{~d} t}+\frac{\varepsilon(t)}{E_{2}} \frac{\mathrm{d}^{2} E_{2}}{\mathrm{~d} t^{2}}\right)$

In the above formula, the left side is the function of the space variable $z$, and the right side is the function of the time variable, and to make two sides be equaled, this formula must equal constant, $-k^{2}$, $\frac{\mathrm{d}^{2} E_{1}}{\mathrm{~d} z^{2}}+k^{2} E_{1}=0$ 


$$
\frac{\mathrm{d}^{2} E_{2}}{\mathrm{~d} t^{2}}+\left(2 \frac{1}{\varepsilon(t)} \frac{\mathrm{d} \varepsilon(t)}{\mathrm{d} t}+\mu_{0} c^{2} \frac{\sigma(t)}{\varepsilon(t)}\right) \frac{\mathrm{d} E_{2}}{\mathrm{~d} t}+\left(\frac{\mu_{0} c^{2}}{\varepsilon(t)} \frac{\mathrm{d} \sigma(t)}{\mathrm{d} t}+\frac{1}{\varepsilon(t)} \frac{\mathrm{d}^{2} \varepsilon(t)}{\mathrm{d} t^{2}}+\frac{k^{2} c^{2}}{\varepsilon(t)}\right) E_{2}=0
$$

To make the above formula have the analytic solution, without loss of generality, take $\varepsilon(t)=a_{0} \exp \left(a_{1} t\right)$ and $\sigma(t)=a_{2} \exp \left(a_{1} t\right)$, and suppose $b_{1}=a_{1}+a_{2} \mu_{0} c^{2} / a_{0}$, and the above equation set can be modified as

$$
\begin{aligned}
& \frac{d^{2} E_{1}}{d z^{2}}+k^{2} E_{1}=0 \\
& \frac{\mathrm{d}^{2} E_{2}}{\mathrm{~d} t^{2}}+\left(a_{1}+b_{1}\right) \frac{\mathrm{d} E_{2}}{\mathrm{~d} t}+\left(a_{1} b_{1}+\frac{k^{2} c^{2}}{a_{0}} \exp \left(-a_{1} t\right)\right) E_{2}=0
\end{aligned}
$$

, and the solution of the equation (10a) is $E_{1}(z)=\exp (\mathrm{i} k z)$, and $k$ can be positive or negative. Supposed that $v=b_{1} / a_{1}-1$, the solution of the equation (10b) is

$$
E_{2}(t)=C_{1} H_{v}^{(1)}\left(\frac{2 k c}{a_{1} \sqrt{a_{0}}} \exp \left(-\frac{a_{1}}{2} t\right)+C_{2} H_{v}^{(2)}\left(\frac{2 k c}{a_{1} \sqrt{a_{0}}} \exp \left(-\frac{a_{1}}{2} t\right)\right)\right.
$$

, where, $C_{1}$ and $C_{2}$ are undetermined coefficients, $H_{v}^{(1)}(x)$ and $H_{v}^{(2)}(x)$ respectively are the V-order first-kind Hank function and the V-order second-kind Hank function. The solution of the equation (9) is

$$
E_{x}^{T}=\exp \left(-\frac{a_{1}+b_{1}}{2} t\right) \times \int_{-\infty}^{+\infty}\left[C _ { 1 } H _ { v } ^ { ( 1 ) } \left(\frac{2 k c}{a_{1} \sqrt{a_{0}}} \exp \left(-\frac{a_{1}}{2} t\right)+C_{2} H_{v}^{(2)}\left(\frac{2 k c}{a_{1} \sqrt{a_{0}}} \exp \left(-\frac{a_{1}}{2} t\right)\right] \exp (\mathrm{i} k z) \mathrm{d} k\right.\right.
$$

Its initial conditions are

$$
\begin{aligned}
& \left.E_{x}^{T}\right|_{t=t_{1}}=E_{0} \exp \left[i\left(\omega t_{1}-k_{0} z\right)\right] \\
& \left.\frac{d E_{x}^{T}}{d t}\right|_{t=t_{1}}=i \omega E_{0} \exp \left[i\left(\omega t_{1}-k_{0} z\right)\right]
\end{aligned}
$$

Supposed that the Fourier conversions of $\left.E_{x}^{T}\right|_{t=t_{1}}$ and $\left.\frac{d E_{x}^{T}}{d t}\right|_{t=t_{1}}$ respectively are $\bar{\varphi}\left(t_{1}, k\right)$ and $\bar{\psi}\left(t_{1}, k\right)$.

For the formula (11), from the initial conditions, the unknown coefficients $C_{1}$ and $C_{2}$ can be solved as follows (the solving process can be used for the reference from the Yang' article (Y. Zhang, 2005, P. 446-449)).

$$
\begin{aligned}
& C_{1}=\frac{\sqrt{a_{0}}}{k c} \exp \left(\left(a_{1}+\frac{b_{1}}{2}\right) t_{1}\right) \times \frac{\left.\bar{\phi}\left(t_{1}, k\right)\left[\left(a_{1}+b_{1}\right) F_{N}+k c \exp \left(-a_{1} t / 2\right) F_{N}^{\prime} / \sqrt{a_{0}}\right)\right]}{F_{J} F_{N}^{\prime}-F_{N} F_{J}^{\prime}}+\frac{\bar{\psi}\left(t_{1}, k\right) F_{N}}{F_{J} F_{N}^{\prime}-F_{N} F_{J}^{\prime}} \\
& C_{2}=-\frac{\sqrt{a_{0}}}{k c} \exp \left(\left(a_{1}+\frac{b_{1}}{2}\right) t_{1}\right) \times \frac{\bar{\phi}\left(t_{1}, k\right)\left[\left(a_{1}+b_{1}\right) F_{J}+k c \exp \left(-a_{1} t / 2\right) F_{J}^{\prime} / \sqrt{a_{0}}\right)}{F_{J} F_{N}^{\prime}-F_{N} F_{J}^{\prime}}+\frac{\bar{\psi}\left(t_{1}, k\right) F_{J}}{F_{J} F_{N}^{\prime}-F_{N} F_{J}^{\prime}}
\end{aligned}
$$

, where,

$$
\begin{aligned}
& F_{J}=H_{v}^{(1)}\left(2 k c \exp \left(-a_{1} t_{1} / 2\right) /\left(a_{1} \sqrt{a_{0}}\right)\right), F_{N}=H_{v}^{(2)}\left(2 k c \exp \left(-a_{1} t_{1} / 2\right) /\left(a_{1} \sqrt{a_{0}}\right)\right), \\
& F_{J}^{\prime}=H_{v}^{(1)}{ }^{\prime}\left(2 k c \exp \left(-a_{1} t_{1} / 2\right) /\left(a_{1} \sqrt{a_{0}}\right)\right), F_{N}^{\prime}=H_{v}^{(2)}{ }^{\prime}\left(2 k c \exp \left(-a_{1} t_{1} / 2\right) /\left(a_{1} \sqrt{a_{0}}\right)\right) .
\end{aligned}
$$

By substituting the expressions of $C_{1}$ and $C_{2}$ into the formula (11), the expression of the forward wave can be denoted as

$$
\begin{aligned}
& E_{1}^{T}=-\exp \left(-\frac{a_{1}+b_{1}}{2} t\right) \frac{\sqrt{a_{0}}}{k_{0} c} \times \exp \left(\left(a_{1}+\frac{b_{1}}{2}\right) t_{1}\right) E_{0} \exp \left(i \omega t_{1}\right) \times \\
& {\left[\frac{\left(a_{1}+b_{1}\right) F_{N 0} / 2+i \omega F_{N 0}}{F_{J 0} F_{N 0}^{\prime}-F_{N 0} F_{J 0}^{\prime}}-\frac{k_{0} c \exp \left(-a_{1} t_{1} / 2\right) F_{N 0}^{\prime} / \sqrt{a_{0}}}{F_{J 0} F_{N 0}^{\prime}-F_{N 0} F_{J 0}^{\prime}}\right] \times H_{v}^{(1)}\left(-\frac{2 k_{0} c}{a_{1} \sqrt{a_{0}}} \exp \left(-\frac{a_{1}}{2} t\right)\right) \exp \left(-i k_{0} z\right)}
\end{aligned}
$$

The expression of the backward wave is 


$$
\begin{aligned}
& E_{2}^{T}=-\exp \left(-\frac{a_{1}+b_{1}}{2} t\right) \frac{\sqrt{a_{0}}}{k_{0} c} \times \exp \left(\left(a_{1}+\frac{b_{1}}{2}\right) t_{1}\right) E_{0} \exp \left(i \omega t_{1}\right) \times \\
& {\left[\frac{\left(a_{1}+b_{1}\right) F_{J 0} / 2+i \omega F_{J 0}}{F_{J 0} F_{N 0}^{\prime}-F_{N 0} F_{J 0}^{\prime}}-\frac{k_{0} c \exp \left(-a_{1} t_{1} / 2\right) F_{J 0}^{\prime} / \sqrt{a_{0}}}{F_{J 0} F_{N 0}^{\prime}-F_{N 0} F_{J 0}^{\prime}}\right] \times H_{v}^{(2)}\left(-\frac{2 k_{0} c}{a_{1} \sqrt{a_{0}}} \exp \left(-\frac{a_{1}}{2} t\right)\right) \exp \left(-k_{0} z\right)}
\end{aligned}
$$

In the above formula,

$$
\begin{aligned}
& F_{J 0}=H_{v}^{(1)}\left(-2 k_{0} c \exp \left(-a_{1} t_{1} / 2\right) /\left(a_{1} \sqrt{a_{0}}\right)\right), F_{N 0}=H_{v}^{(2)}\left(-2 k_{0} c \exp \left(-a_{1} t_{1} / 2\right) /\left(a_{1} \sqrt{a_{0}}\right)\right), \\
& F_{J 0}^{\prime}=H_{v}^{(1)}\left(-2 k_{0} c \exp \left(-a_{1} t_{1} / 2\right) /\left(a_{1} \sqrt{a_{0}}\right)\right), F_{N 0}^{\prime}=H_{v}^{(2) '}\left(-2 k_{0} c \exp \left(-a_{1} t_{1} / 2\right) /\left(a_{1} \sqrt{a_{0}}\right)\right) .
\end{aligned}
$$

The complete expression of the electric-field component $E_{x}^{T}$ in the time-varying medium is

$$
E_{x}^{T}=E_{1}^{T}+E_{2}^{T}
$$

The formula (12) is the expression of the electric-field component in the time-varying medium with the conditions including $\varepsilon(t)=a_{0} \exp \left(a_{1} t\right)$ and $\sigma(t)=a_{2} \exp \left(a_{1} t\right)$. According to R. F. Harrington' article (R. F. Harrington, 2001), the gradual formula of $H_{v}^{(1)}(x)$ has the factor $\exp (\mathrm{i} x)$, and the gradual formula of $H_{v}^{(2)}$ has the factor $\exp (-\mathrm{i} x)$, so the first item of the formula (12) in the left of the equal sign denotes the wave propagating along the direction of $+\mathrm{z}$ in the Area Two, and the second item denotes the wave propagating along the direction of $-\mathrm{z}$ in the Area Two. For other time-varying forms of the medium, the analytic solutions can be solved in the same way, and if the analytic solutions cannot be solved, the method of the numerical iteration can be used.

For the wave $E_{2}^{T}$ propagating along the direction of $-\mathrm{z}$ in the Area Two, the method of taking limit for time-varying increment can be used to solve the reflection and transmission coefficients on the interface in the Area One and the Area Two. The transient reflection coefficient that the reflection wave (propagating along the direction of $-\mathrm{z}$ ) at the time of $t$ in the Area Two propagating to the interface and the transient propagation coefficient entering into the Area One respectively are

$$
\begin{aligned}
& \Gamma_{1}(t)=\lim _{\Delta t \rightarrow 0} \Gamma_{1}(t+\Delta t / 2)=\frac{\eta_{0}-\eta_{1}(t)}{\eta_{0}+\eta_{1}(t)} \\
& T_{1}(t)=\lim _{\Delta t \rightarrow 0} T_{1}(t+\Delta t / 2)=\frac{2 \eta_{0}}{\eta_{0}+\eta_{1}(t)}
\end{aligned}
$$

\section{Test of the solution validity}

The correctness of the expression (12) about the electric-field intensity in the time-varying medium can be tested as follows.

First, when $a_{0}=1, a_{1}$ and $a_{2}$ are closed to 0 at the same time, and according to the change forms of the time-varying medium, $\varepsilon(t)=a_{0} \exp \left(a_{1} t\right)$ and $\sigma(t)=a_{2} \exp \left(a_{1} t\right)$, and the characteristics of the medium in the Area Two are close to the characteristics of the medium in the Area One. The electric-field component formula (12) of the electromagnetic wave in the Area Two has corresponding changes at this time.

When $x$ is big, such as $x \geq 50$, the first-kind Hank function and the second-kind Hank function have following approximate formulas.

$$
\begin{aligned}
& H_{v}^{(1)}(x) \approx \sqrt{2 / \pi x} \exp [\mathrm{i}(x-v \pi / 2-\pi / 4)] \\
& H_{v}^{(2)}(x) \approx \sqrt{2 / \pi x} \exp [-\mathrm{i}(x-v \pi / 2-\pi / 4)]
\end{aligned}
$$

Substitute them into the formula (12), and

$$
\begin{aligned}
& E_{x}^{T} \approx \exp \left(\frac{a_{1}+b_{1}}{2}\left(t_{1}-t\right)\right) \frac{\sqrt{a_{0}}}{k_{0} c} \exp \left(\frac{a_{1}}{2} t_{1}\right) \times E_{0} \exp \left(\mathrm{i} \omega t_{1}\right)\left(\left[\frac{a_{1}+b_{1}}{2}+\mathrm{i} \omega+\frac{k_{0} c}{\sqrt{a_{0}}} \exp \left(-\frac{a_{1}}{2} t_{1}\right) \times\right.\right. \\
& \left.\left(\mathrm{i}+\frac{a_{1} \sqrt{a_{0}}}{2 k_{0} c} \exp \left(\frac{a_{1}}{2} t_{1}\right) v\right)\right] \frac{1}{2 \mathrm{i}} \exp \left(\frac{a_{1}}{4}\left(t-t_{1}\right)\right) \times \exp \left[\mathrm{i}\left(\frac{2 k_{0} c}{a_{1} \sqrt{a_{0}}}\left(\exp \left(-\frac{a_{1}}{2} t_{1}\right)-\exp \left(-\frac{a_{1}}{2} t\right)\right)-k_{0} z\right)\right]-
\end{aligned}
$$




$$
\begin{aligned}
& {\left[\frac{a_{1}+b_{1}}{2}+\mathrm{i} \omega-\frac{k_{0} c}{\sqrt{a_{0}}} \exp \left(-\frac{a_{1}}{2} t_{1}\right)\left(\mathrm{i}-\frac{a_{1} \sqrt{a_{0}}}{2 k_{0} c} \times \exp \left(\frac{a_{1}}{2} t_{1}\right) v\right)\right] \frac{1}{2 \mathrm{i}} \exp \left(\frac{a_{1}}{4}\left(t-t_{1}\right)\right) \times} \\
& \left.\exp \left[\left(\frac{2 k_{0} c}{a_{1} \sqrt{a_{0}}}\left(\exp \left(-\frac{a_{1}}{2} t\right)-\exp \left(-\frac{a_{1}}{2} t_{1}\right)\right)-k_{0} z\right)\right]\right)
\end{aligned}
$$

When $a_{1}$ and $a_{2}$ are close to 0 at the same time, and $a_{0}=\varepsilon_{0}$, take the limit for the formula (12'),

$$
\lim _{a_{1} \rightarrow 0, a_{2} \rightarrow 0, a_{0} \rightarrow \varepsilon_{0}} E_{x}^{T}=E_{0} \exp \left(i\left(\omega t-k_{0} z\right)\right)
$$

This formula is the expression of the electric-field component of the incident wave. That indicates when the parameters in the time-varying medium are close to the parameters of the time-invariant medium in the Area One, the expression of the electric-field component in the corresponding time-varying medium is close to the expression of the electric-field component in the Area One, which shows that the solution of the formula (12) is correct to some extent, and the time-invariant medium is the special situation of the time-varying medium at the same time (relative to the situation that the relative dielectric constant is the constant). Specially, when $a_{1}$ and $a_{2}$ are close to 0 at the same time, the part of the electric-field intensity of the time-varying medium in the Area Two propagating along the direction of $+\mathrm{z}$ is close to the initial electric-field intensity, and the part of the electric-field intensity propagating along the direction of $-\mathrm{z}$ is close to 0 .

Second, when $a_{0} \neq 1, a_{1}$ and $a_{2}$ are closed to 0 at the same time, the time-varying medium belongs to the mutant type. At this time, the wave propagating along the direction of $+\mathrm{z}$ is split into the forward wave $E_{x f}^{T}$ and the backward wave $E_{x r}^{T}$.

$$
\begin{aligned}
& E_{x f}^{T}=\lim _{a_{1}, a_{2} \rightarrow 0} E_{1}^{T}=\frac{1}{2}\left(\sqrt{a_{0}}+1\right) \times \exp \left[i\left(\frac{\omega}{\sqrt{a_{0}}}\left(t-t_{1}\right)+\omega t_{1}-k_{0} z\right)\right] \\
& E_{x r}^{T}=\lim _{a_{1}, a_{2} \rightarrow 0} E_{2}^{T}=\frac{1}{2}\left(\sqrt{a_{0}}-1\right) \times \exp \left[i\left(\frac{\omega}{\sqrt{a_{0}}}\left(t_{1}-t\right)+\omega t_{1}-k_{0} z\right)\right]
\end{aligned}
$$

The rates between the incident wave with the forward wave and the backward wave when $t=t_{1}$ respectively are

$$
\begin{aligned}
& \frac{E_{x f}^{T}}{E_{x}^{i}}=\frac{1}{2}\left(\sqrt{a_{0}}+1\right) \\
& \frac{E_{x r}^{T}}{E_{x}^{i}}=\frac{1}{2}\left(\sqrt{a_{0}}-1\right)
\end{aligned}
$$

These two expressions are same with the propagation coefficient and the reflection coefficient of the forward wave and the backward wave when the plane wave mutates in the time-varying medium (Ruiz T M, Wright C L, 1978, P. 358-361), which also tests the correctness of the formula (12) from another aspect.

The correctness of the formula (12) can also be tested by the FDTD method. Because $\varepsilon(t)$ and $\sigma(t)$ change with the time, and the iteration formula of the electromagnetic field is not same with the general iterative formula. The concrete forms are

$$
\begin{aligned}
& E_{x}^{n+1}(i)=C A(n) E_{x}^{n}(i)-C B(n) \times \frac{H_{y}^{n+1 / 2}(i+1 / 2)-H_{y}^{n+1 / 2}(i-1 / 2)}{\Delta z} \\
& H_{y}^{n+1 / 2}(i+1 / 2)=H_{y}^{n-1 / 2}(i+1 / 2)-\frac{\Delta t}{\mu_{0}} \times \frac{E_{x}^{n}(i+1)-E_{x}^{n}(i)}{\Delta z}
\end{aligned}
$$

, where,

$$
C B(n)=1 /\left(\frac{\varepsilon_{0} \varepsilon(n+1 / 2)}{\Delta t}-\frac{\varepsilon_{0} \varepsilon^{\prime}(n+1 / 2)+\sigma(n+1 / 2)}{2}\right)
$$




$$
C A(n)=\left(\frac{\varepsilon_{0} \varepsilon(n+1 / 2)}{\Delta t}-\frac{\varepsilon_{0} \varepsilon^{\prime}(n+1 / 2)+\sigma(n+1 / 2)}{2}\right) C B(n)
$$

The boundary conditions of the problem can be described as follows. The left end point is set as the first-order absorption boundary condition, and the right end point is in the region of the time-varying medium, and the values on the interior-layer network points are endowed to the values at the end points, and the comparison of the obtained result and the analytic result is seen in Figure 2 (in the time-varying area) and Figure 7 (the total electromagnetic fields in the time-variant area). Two curve lines in Figure 2 fit well. The numerical results in Figure 7 are largely different with the deviations from the analytic results at the position of peak value, but both change tendencies are consistent as a whole, which indicates the formula (12) is right again. Other aspects will be discussed as follows.

\section{Results and discussion}

As the example, take the frequency of the initial plane wave $f=3.0 \times 10^{9}$, and the amplitude $E_{0}=1 \mathrm{~V} / \mathrm{m}$. The parameters of the medium include $a_{0}=2, a_{1}=2.0 \times 10^{8}$, and $a_{3}=0.001$. The point in the Area One is $z_{1}=-0.1 \mathrm{~m}$, and the point in the Area Two is $z_{2}=1.5 \mathrm{~m}$. For the convenient, $t_{0}=0 \mathrm{~s}$, and according to relative expression, the results are seen in Figure 2 to Figure 7. From these figures, following conclusions can be obtained.

When the electromagnetic wave is propagated in the free space, if the medium in the right half space changes continually at the time of $t_{0}$, the electromagnetic wave will change at the space boundary and at the time boundary. Its physical process can be described as follows.

(1) At the space boundary, i.e. the interface between the time-varying medium and the time-invariant medium, the initial wave is split into two beams, and one beam is the reflection wave, and the other beam is the transmission wave from the interface to the time-varying medium, and these two beams of wave have same frequency with the initial electromagnetic wave, but both the reflection wave and the initial wave have same wave number, and because the time-varying medium has different medium parameters at different times, the transmission wave has different wave numbers at different times, which can be seen in Figure 6 .

(2) Because the parameters of the medium in the Area Two change with the time, the reflection coefficients on the boundary and the propagation coefficients entering into the Area Two will also change with the time (seen in Figure 4 and Figure 5).

(3) At the moment that the wave propagating in the right half space begins to change, the wave is split into two beams, and one beam is the forward wave, and the other beam is the backward wave, and the wave combined by these two beams is seen in Figure 2, and the backward wave is seen in Figure 3, and their frequencies are not same with the frequency of the initial wave, but the frequencies changing with the time. From Figure 2 and Figure 3, when the relative dielectric constant $\varepsilon(t)$ increases with the time exponent, the amplitude of the electric-field intensity in the time-varying medium will decrease gradually, and the cycle increases gradually with the time, and the amplitude of the backward wave is far less than the amplitude of the forward wave.

(4) The wave propagating backward in the Area Two will also reflect and transmit at the interface between the time-varying medium and the time-invariant medium, and the corresponding reflection coefficient and the propagation coefficient are the functions about time, and because the amplitude of the backward wave is less than the amplitude of the forward wave, the quantity transmitting into the Area One is smaller relative to the initial wave. The figure that the incident wave in the Area one superposes with the reflection wave at the boundary at the position of $z=-0.1 \mathrm{~m}$ is seen in Figure 7. The frequency of the incident wave is same with the frequency of the reflection wave, but the frequency of the transmission wave from the time-varying medium area changes. Because the amplitude is small, the influence on the frequency of the total electromagnetic field is small. The amplitude of the synthesis wave changes with the time, because of the superposition of these three waves.

The above result is based on the situation of the parameter $a_{1}>0$, and in the same way, the situation of $a_{1}<0$ can also be discussed. Specially, this only shows a kind of time-varying situation, and other changes can be solved by the analyzing method or the numerical method, which will be discussed in the future.

\section{References}

A.G. Nerukh, P. Sewell and T. M. Benson. (2004). Volterra integral equations for nonstationary electromagnetic processes in time-varying dielectric waveguides. Journal of Lightwave Technology. No. 22 (5). P. 1408-1419. 
B. K. Chen, B. Q. Gao. (2009). Accurate Solution and Characteristics for Electromagnetic Wave Propagation in Time-varying Medium. Modern Applied Science. No. 3. P. 68-76.

B. K. Chen, Y. Zhang and B.Q. Gao. (2006). Solution of electromagnetic wave in time-varying medium in two-dimensional space. Chin. Phys. Lett. No. 23(1). P. 595-598.

F. R. Morenthaler. (1958). Velocity modulation of electromagnetic waves. IRE Trans. Microwave Theory Tech. No. 6(4). P. 167-172.

Ouyang, Ziyuan. (2005). General Introduction of Lunar Science. Beijing: China Aerospace Press.

Ren, Wu \& Gao, Benqing. (2003). The Analysis of Effects in Time-varying Medium by FDTD Method. Chinese Journal of Radio Science. No. 18(1). P. 18-22.

R. F. Harrington. (2001). Time-Harmonic Electro-magnetic Fields. New York: IEEE Press.

R. L. Fante. (1971). Transmission of electromagnetic waves into time-varying medium. IEEE Trans Antennas Propagat. No. 19(5). P. 417-424.

Ruiz T M, Wright C L. (1978). Characteristics of electromagnetic wave propagating in time-varying medium. IEEE Trans Antennas Propagat. No. 26 (2). P. 358-361.

Y. Zhang and B. Q. Gao. (2005). Propagation of cylindrical waves in medium of time-dependent permittivity. Chin. Phys. Lett. No. 22 (2). P. 446-449.

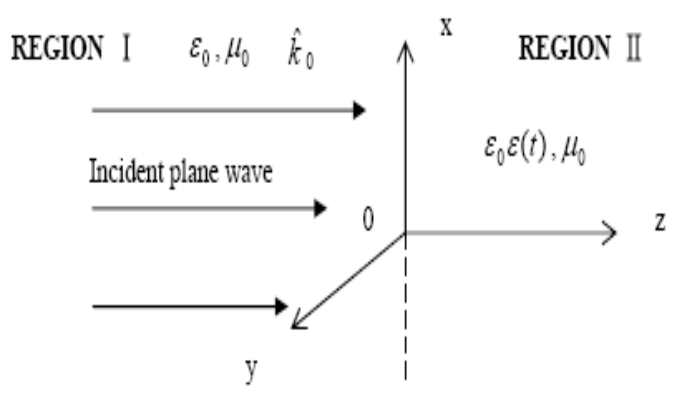

Figure 1. Diagram of Problem $\left(t>t_{1}\right)$

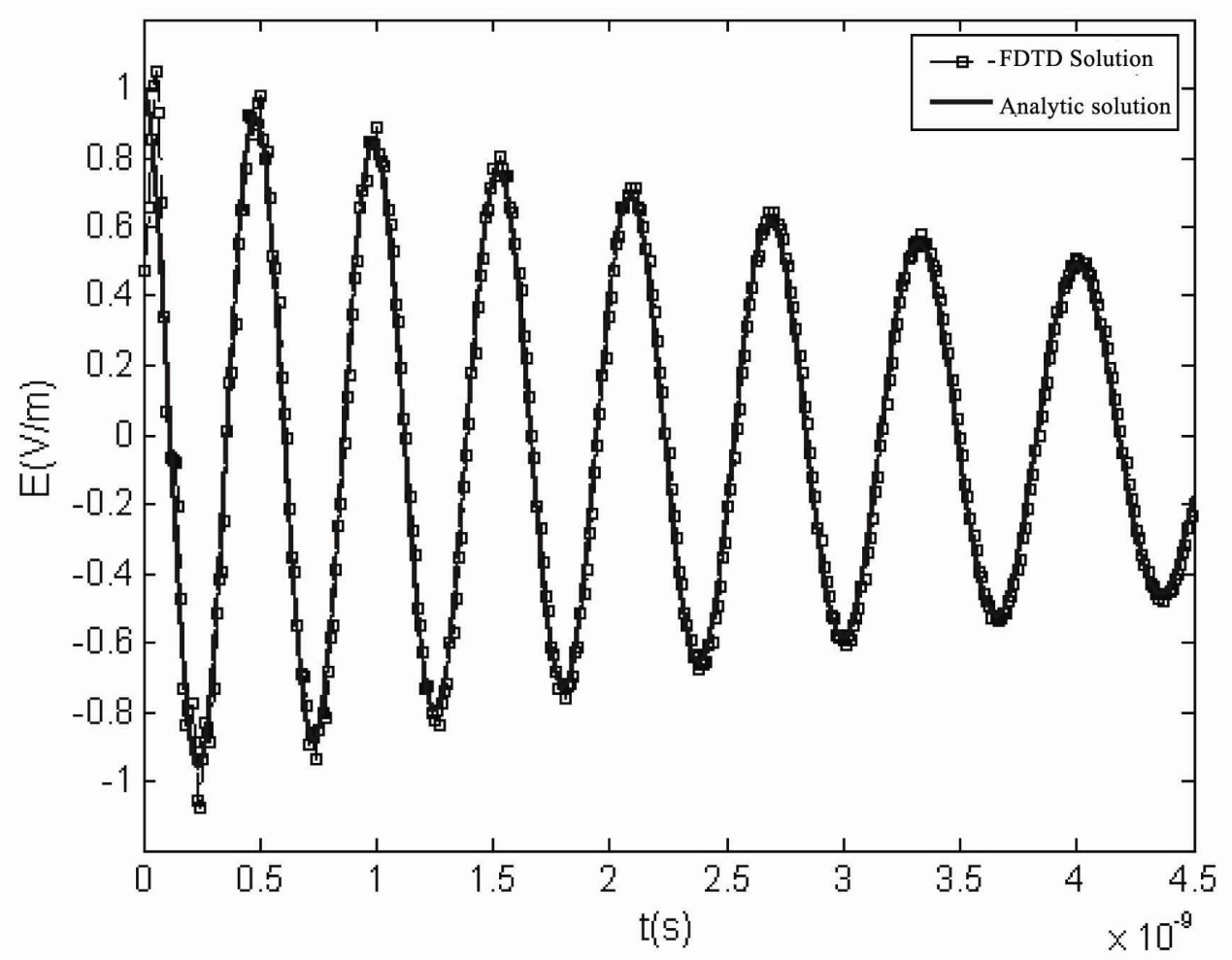

Figure 2. Change of the Total Electric Intensity Propagated at $\mathrm{z}=1.5 \mathrm{~m}$ in Time-varying Medium with time

$$
\left(a_{1}>0\right)
$$




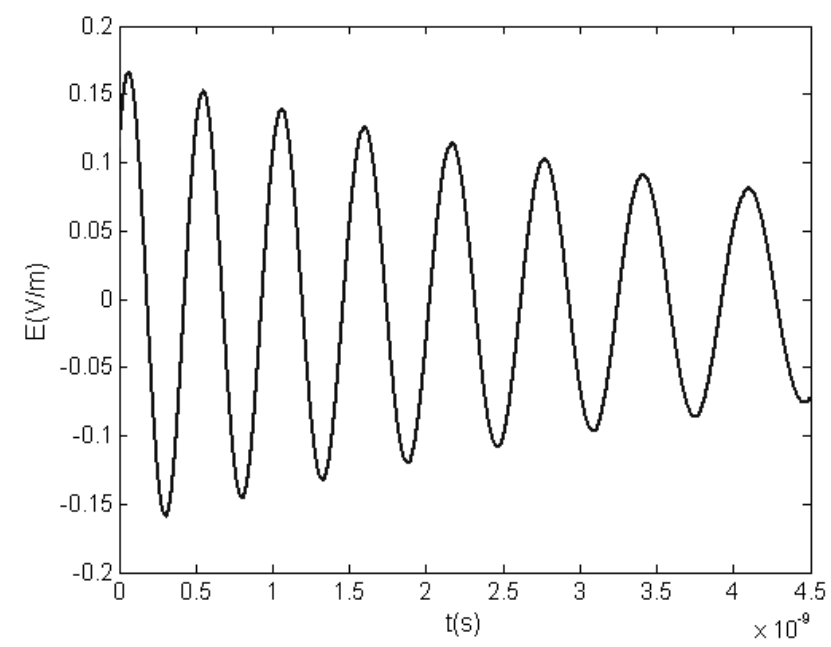

Figure 3. Change of the Electric Intensity of the Electromagnetic Wave at $\mathrm{z}=1.5 \mathrm{~m}$ Propagating along the Direction of $\mathrm{Z}$ in Time-varying Medium with time $\left(a_{1}>0\right)$

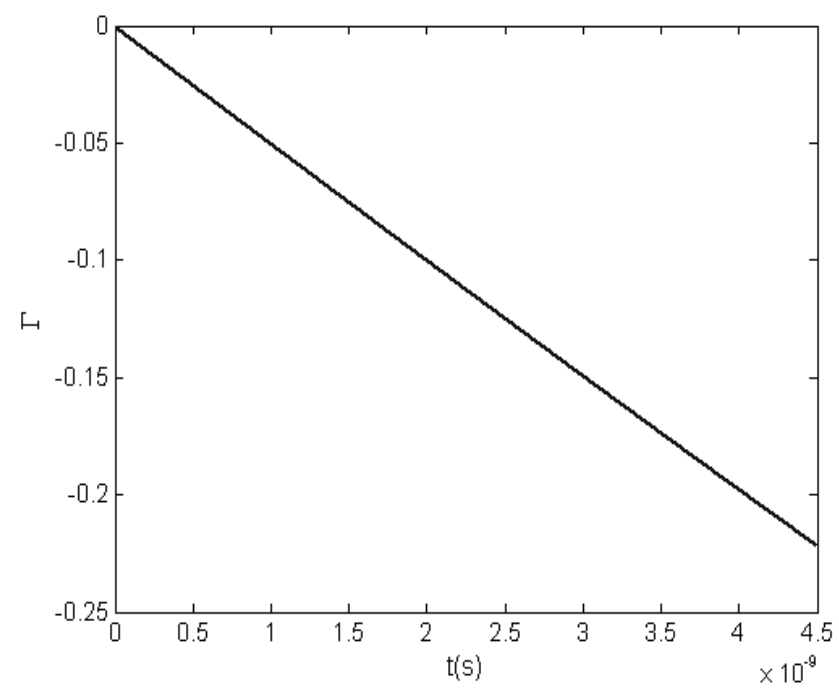

Figure 4. Change of the Reflection Coefficient of the Initial Incident Wave on the Interface between the Time-varying Medium and the Time-invariant Medium

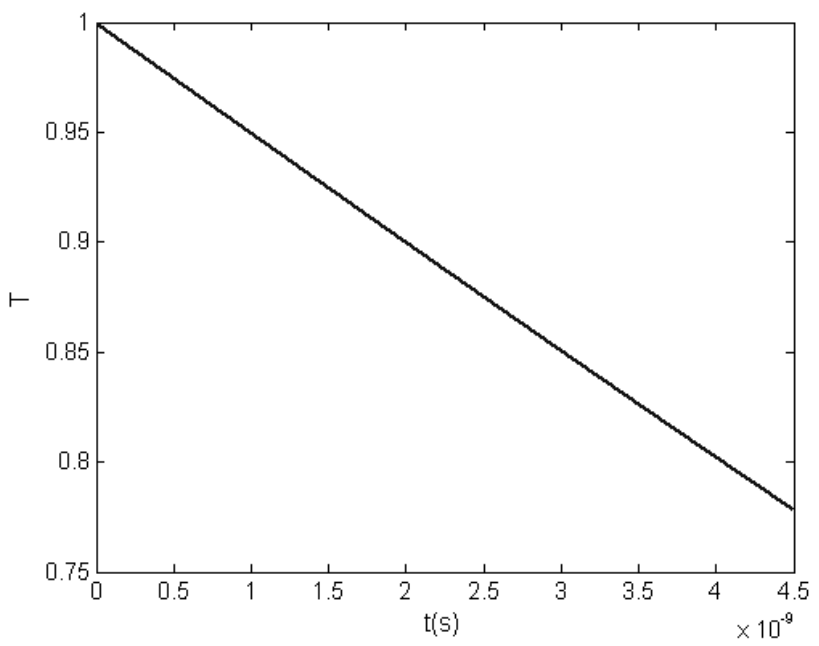

Figure 5. Change of the Propagation Coefficient When the Electromagnetic Wave Enters in the Area Two on the Interface between the Time-varying Medium and the Time-invariant Medium 


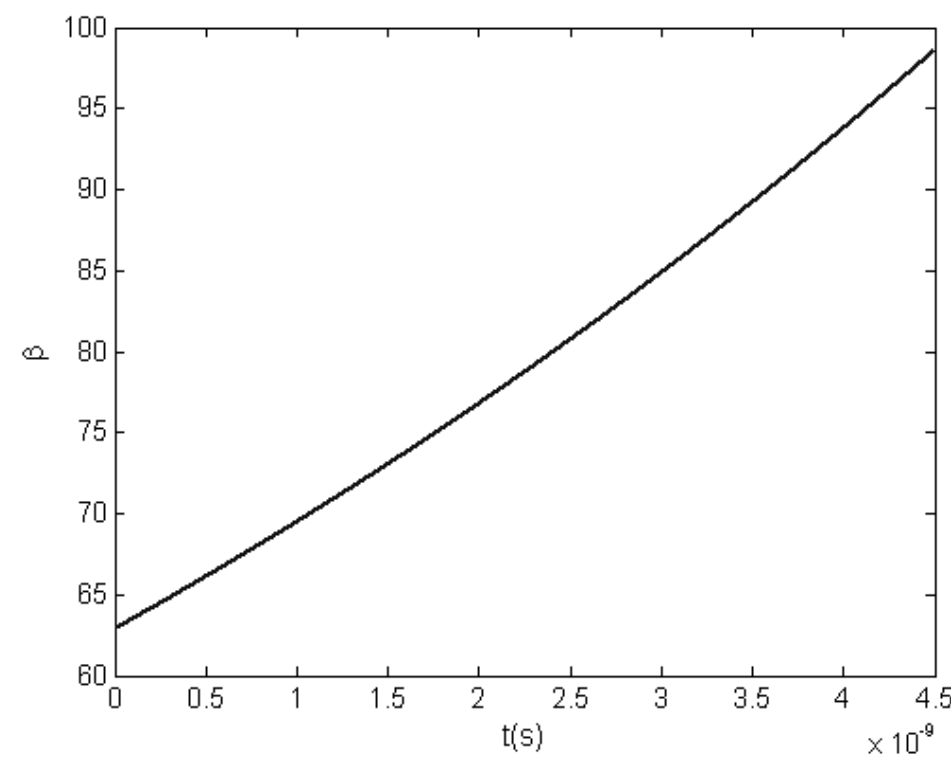

Figure 6. Change of the Propagation Constant of the Electromagnetic Wave Enters in the Area of $z>0$ When the Electromagnetic Wave Enters into the Time-varying Medium from the Time-invariant Medium at Different Moments

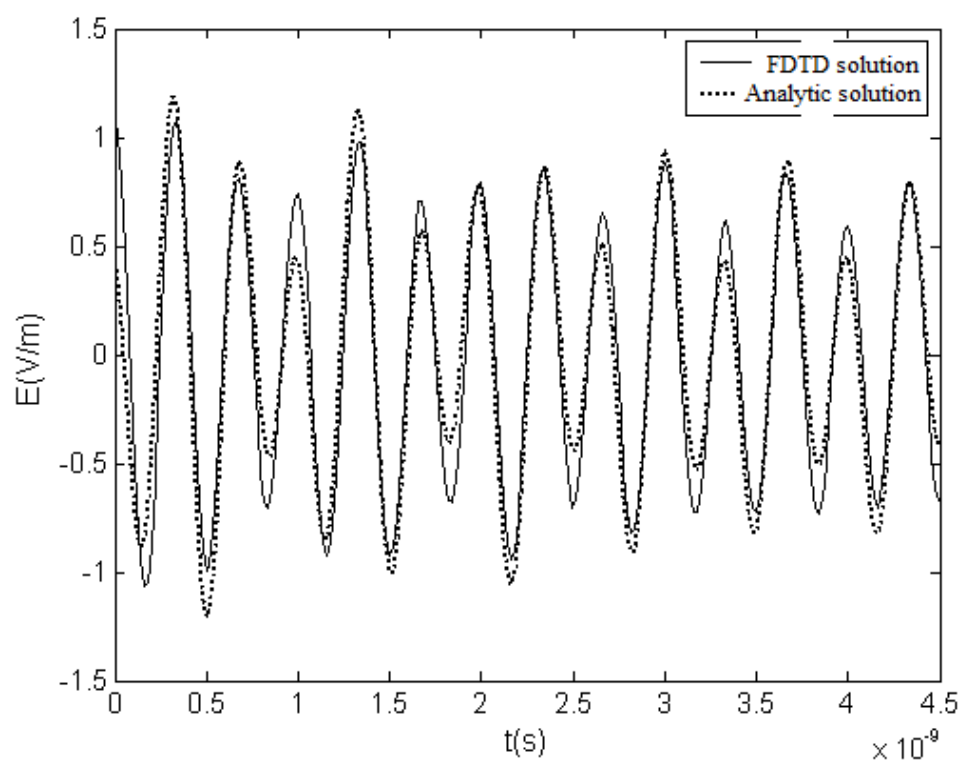

Figure 7. Total Electric Intensity at $\mathrm{z}=-0.1 \mathrm{~m}$ Superposed by the Incident Wave in the Area One, the Interface Wave, and the Transmitted Wave from the Area Two 